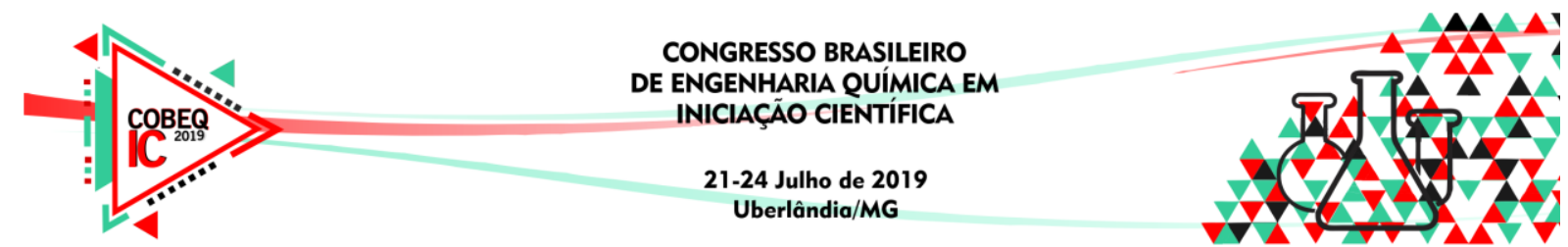

\title{
OBTENÇÃO DE ACETATO DE CELULOSE VIA ACETILAÇÃO HOMOGÊNEA A PARTIR DE CELULOSE BRANQUEADA DE BAGAÇO DE CANA
}

\author{
S. A. Y. KAWASHIMA ${ }^{1}$, G. O. L. SANTOS ${ }^{1}$, E.J.C MORAES ${ }^{1}$ e L. F. F. FARIA ${ }^{1}$ \\ ${ }^{1}$ Universidade de São Paulo, Departamento de Engenharia Química \\ E-mail para contato: figue@ usp.br
}

\begin{abstract}
RESUMO - O objetivo foi o estudo da obtenção de acetato de celulose (AC) a partir da celulose de bagaço de cana-de-açúcar, o qual foi submetido a uma sequência de tratamentos químicos, a fim de se obter uma polpa celulósica mais purificada. Para obtenção de AC foi utilizado o método de acetilação homogênea. O estudo desta síntese foi realizado através de um planejamento experimental exploratório do tipo Fatorial Completo, em que as variáveis de controle estudadas foram os teores de anidrido acético, ácido acético e ácido sulfúrico. Como variáveis resposta, foram avaliadas a eficiência de obtenção e o grau de substituição (GS). Após análises estatísticas, foi observado que os fatores mais influentes para ambas as variáveis resposta foram o teor de anidrido acético e ácido sulfúrico. $\mathrm{O}$ ensaio que apresentou melhor resultado foi realizado contendo $64 \mathrm{~mL}$ de anidrido acético, $20 \mathrm{~mL}$ de ácido acético e $0,1 \mathrm{~mL}$ de ácido sulfúrico, proporcionando uma eficiência de $81,2 \%$ e GS $=2,88$.
\end{abstract}

\section{INTRODUÇÃO}

O Brasil é um dos maiores produtores de cana-de-açúcar do mundo, cuja safra 2018/2019 tem previsão de 626 milhões de toneladas (CONAB, 2018). Uma tonelada de colmo de cana contém cerca de $130 \mathrm{~kg}$ de bagaço e, apesar de grande parte deste ser utilizado na geração de energia renovável (SEABRA, 2018), ainda há muito excedente. O bagaço de cana é composto, principalmente, por cerca de 40 a $50 \%$ de celulose, 25 a $35 \%$ de hemicelulose e 15 a $25 \%$ de lignina (ARISTIDOU; PENTTILÄ, 2000). Um dos principais biopolímeros é a celulose, abundante e de ampla utilidade. Porém, devido às propriedades de suas ligações químicas, é insolúvel em água, limitando sua processabilidade.

Para contornar essa limitação quanto a solubilidade, são usados derivados químicos de celulose. A conversão da celulose em ésteres de celulose produz materiais que podem ser solubilizados ou processados no estado fundido. Dentre vários ésteres existentes atualmente, o $\mathrm{AC}$ e os ésteres mistos (propianato acetato de celulose e butirato acetato de celulose) são os mais importantes comercial e industrialmente, devido suas propriedades e fácil manuseio.

A produção de AC ocorre pela substituição dos grupos hidroxilas das unidades de glicose por grupos acetila. Dessa forma, diferentes GS podem ser obtidos através da esterificação da celulose, sendo o máximo igual a três (PULEO et al., 1989). O GS de um polímero está diretamente relacionado com características como solubilidade, cristalinidade, 


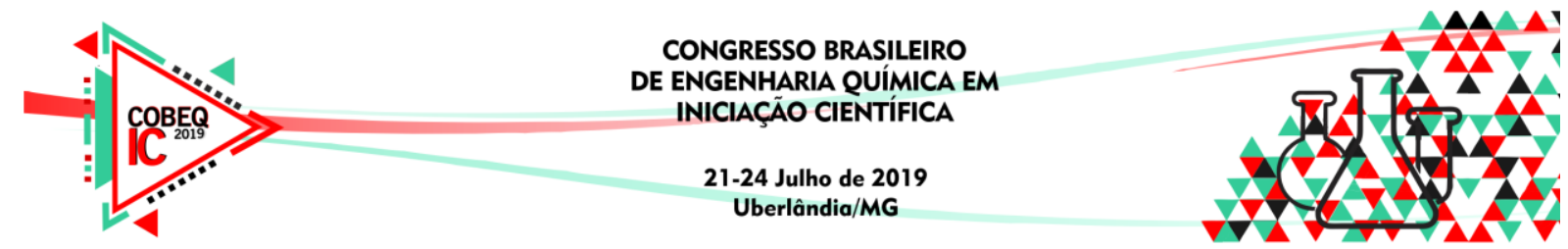

biodegradabilidade, entre outras. O AC mais utilizado comercialmente é o secundário, que possui uma média de 2,5 grupos hidroxilas substituídos por unidade de glicose. Triacetatos possuem GS maior que 2,7 (ZUGENMAIER, 2004), sendo que o comercial apresenta em média GS = 2,88 (CANDIDO, 2011). Esses materiais são usados em filmes plásticos, revestimentos superficiais, produção de membranas, entre outros (CHENG et al, 2010).

Assim, devido às características e ao grande interesse comercial existente, um estudo mais aprofundado das condições reacionais da síntese de acetato de celulose, a fim de otimizar sua produção, é de suma importância para que se possa obter produtos de qualidade e ambientalmente amigáveis, de modo a agregar valor ao bagaço de cana.

\section{METODOLOGIA}

\subsection{Obtenção de Acetato de Celulose}

O AC foi obtido baseado na metodologia de Ferreira Júnior et al. (2010). A partir da purificação e branqueamento $2 \mathrm{~g}$ de celulose foram misturados com ácido acético e agitados por 30 minutos. A seguir, ácido sulfúrico e $27 \mathrm{~mL}$ de ácido acético foram adicionados ao sistema, que ficou sob agitação por 25 minutos. A mistura foi filtrada e, ao filtrado, foi adicionado anidrido acético. A solução foi retornada ao recipiente contendo celulose e agitada por $30 \mathrm{~min}$. Passado esse tempo, a mistura ficou em repouso por $15 \mathrm{~h}$ e, depois disso, foi precipitada em meio aquoso. Por fim, o AC foi filtrado e seco, à $50^{\circ} \mathrm{C}$.

Planejamento de experimentos para otimização da obtenção de AC: Os experimentos foram realizados a partir de um planejamento do tipo Fatorial Completo $2^{3}$, com triplicata no ponto central. As variáveis controle foram os teores de ácido acético, anidrido acético e ácido sulfúrico. Os níveis das variáveis estão dispostos na Tabela 1. As análises estatísticas foram realizadas com o apoio do Software Minitab® 18.

Tabela 1 - Variáveis controle e níveis do planejamento fatorial $2^{3}$

\begin{tabular}{cccc}
\hline \multirow{2}{*}{ Variável controle } & \multicolumn{3}{c}{ Níveis } \\
\cline { 2 - 4 } & Baixo (-1) & Central (0) & Alto (+1) \\
\hline $\mathbf{C H}_{\mathbf{3}} \mathbf{C O O H}(\mathbf{m L})$ & 20 & 35 & 50 \\
$\mathbf{C}_{\mathbf{4}} \mathbf{H}_{\mathbf{6}} \mathbf{O}_{\mathbf{3}}(\mathbf{m L})$ & 20 & 42 & 64 \\
$\mathbf{H}_{\mathbf{2}} \mathbf{S O}_{\mathbf{4}}(\mathbf{m L})$ & 0,10 & 0,13 & 0,16 \\
\hline
\end{tabular}

Determinação do GS: Em 0,1g de triacetato de celulose foram adicionados 5,0mL de $\mathrm{NaOH}(0,25 \mathrm{M})$ e $5,0 \mathrm{~mL}$ de etanol. Depois de $24 \mathrm{~h}$, foram adicionados ao sistema $10,0 \mathrm{~mL}$ de $\mathrm{HCl}(0,25 \mathrm{M})$. Após $30 \mathrm{~min}$, a mistura foi titulada com $\mathrm{NaOH}$, padronizado previamente com biftalato de potássio e, como indicador, foi usado fenolftaleína. Assim, a porcentagem de grupos acetil (\%GA) foi determinada de acordo com Ferreira Júnior et al. (2010). Sabendo que o triacetato de celulose comercial possui, em média, um GS $=2,88$ correspondente a $43,5 \%$ de grupos acetil, o GS foi determinado por proporcionalidade.

Determinação da eficiência de obtenção do AC: A determinação da eficiência de obtenção do AC consiste em um parâmetro de normalização comparativo com o grau máximo teórico de conversão de celulose em AC, considerando a acetilação total de todas as hidroxilas como o valor de máxima eficiência, conforme a Equação 1. 


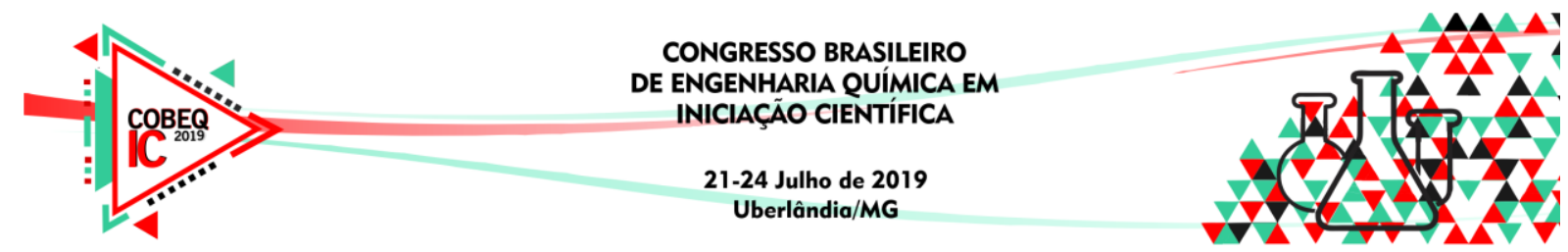

$$
\mathrm{Ef}=\frac{\mathrm{m}_{\mathrm{ac}}{ }^{*} 100}{\mathrm{M}^{* 1,818}}
$$

Onde: $\mathrm{Ef}=$ eficiência; $\mathrm{m}_{\mathrm{ac}}=$ massa de triacetato de celulose $; \mathrm{M}=$ massa seca inicial de celulose branqueada.

\section{RESULTADOS E DISCUSSÃO}

A Tabela 2 apresenta a matriz experimental proposta para as variáveis controle, assim como os resultados obtidos para ambas variáveis resposta.

Tabela 2 - Matriz experimental proposta e respectivos valores para as variáveis resposta eficiência e GS.

\begin{tabular}{cccc|cc}
\hline Ensaios & $\begin{array}{c}\text { Teor de } \mathrm{C}_{4} \mathrm{H}_{6} \mathrm{O}_{3} \\
(\mathbf{m L})\end{array}$ & $\begin{array}{c}\text { Teor de } \mathrm{CH}_{3} \mathrm{COOH} \\
(\mathbf{m L})\end{array}$ & $\begin{array}{c}\text { Teor de } \mathrm{H}_{2} \mathrm{SO}_{4} \\
(\mathbf{m L})\end{array}$ & $\begin{array}{c}\text { Eficiência } \\
(\boldsymbol{\%})\end{array}$ & GS \\
\hline $\mathbf{1}$ & 20 & 20 & 0,10 & 4,3 & 2,74 \\
$\mathbf{2}$ & 64 & 20 & 0,10 & 81,2 & 2,88 \\
$\mathbf{3}$ & 20 & 50 & 0,10 & $\mathrm{Nd}$ & $\mathrm{Nd}$ \\
$\mathbf{4}$ & 64 & 50 & 0,10 & 59,6 & 2,79 \\
$\mathbf{5}$ & 20 & 20 & 0,16 & 4,3 & 2,70 \\
$\mathbf{6}$ & 64 & 20 & 0,16 & 71,2 & 2,43 \\
$\mathbf{7}$ & 20 & 50 & 0,16 & $\mathrm{Nd}$ & $\mathrm{Nd}$ \\
$\mathbf{8}$ & 64 & 50 & 0,16 & 94,4 & 2,70 \\
$\mathbf{9}$ & 42 & 35 & 0,13 & 73,6 & 2,57 \\
$\mathbf{1 0}$ & 42 & 35 & 0,13 & 50,9 & 2,56 \\
$\mathbf{1 1}$ & 42 & 35 & 0,13 & 75,9 & 2,79 \\
\hline
\end{tabular}

- $\mathrm{Nd}$ - Não detectável

A Figura 1 apresenta os gráficos de Pareto e efeitos principais individualizados para a variável resposta eficiência. Através da análise do gráfico de Pareto, percebe-se que, embora nenhuma variável controle tenha sido considerada significativa a um nível de confiança de 95\%, o teor de anidrido acético combinado com o teor de ácido sulfúrico apresentou maior influência nos resultados, enquanto o teor de ácido acético isolado apresentou comportamento contrário.

Figura 1 - Gráficos de Pareto e efeitos principais para a variável resposta eficiência.

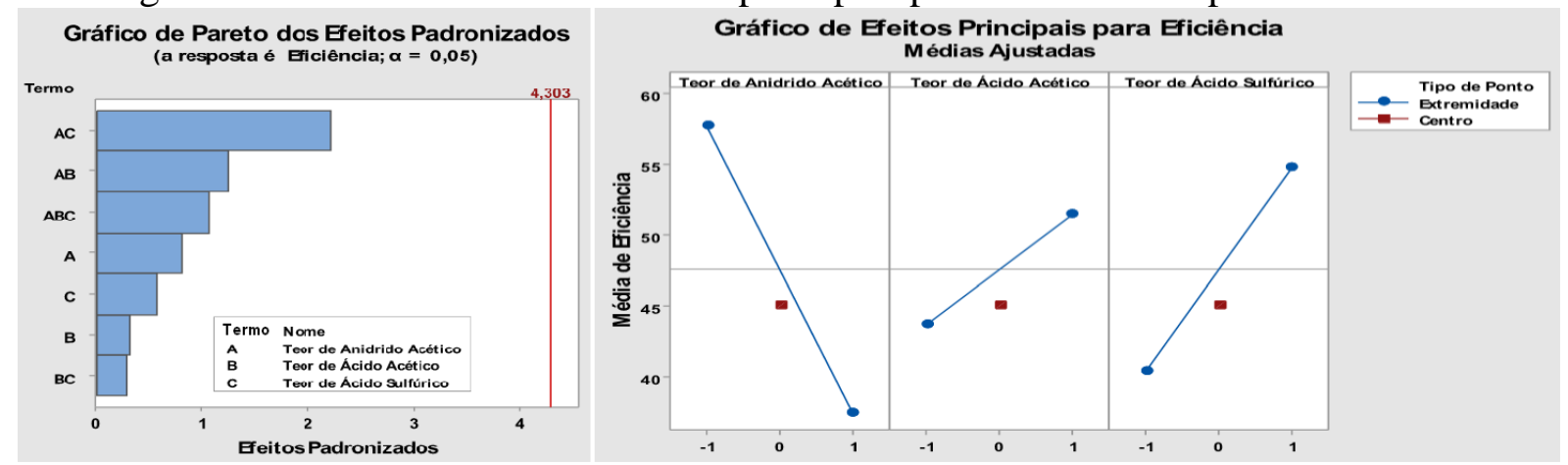




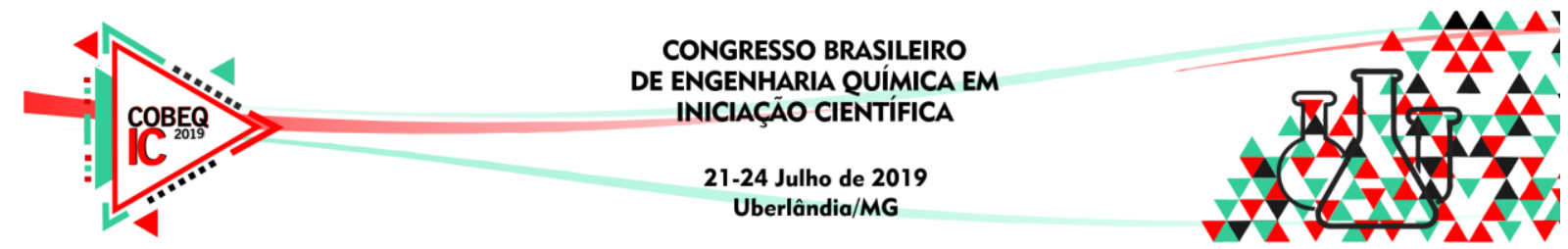

A análise dos efeitos principais individualizados permite observar claramente a maior importância do teor de anidrido acético, seguido pelo teor de ácido sulfúrico. Contudo, destaca-se a influência contrária com relação aos níveis, uma vez que teores elevados de anidrido na presença de valores reduzidos de ambos os ácidos tendem a apresentar melhores resultados.

Pela análise do diagrama de Pareto e dos efeitos individualizados para a variável resposta GS (Figuras 2), pode-se observar que as variáveis que apresentaram maior significância estatística foram os teores de anidrido acético e ácido sulfúrico, através da ação combinada de ambas. Isto permite concluir que os efeitos sinérgicos entre as variáveis são extremamente atuantes nas condições avaliadas.

Figura 2 - Diagrama de Pareto e efeitos principais para a variável resposta GS.
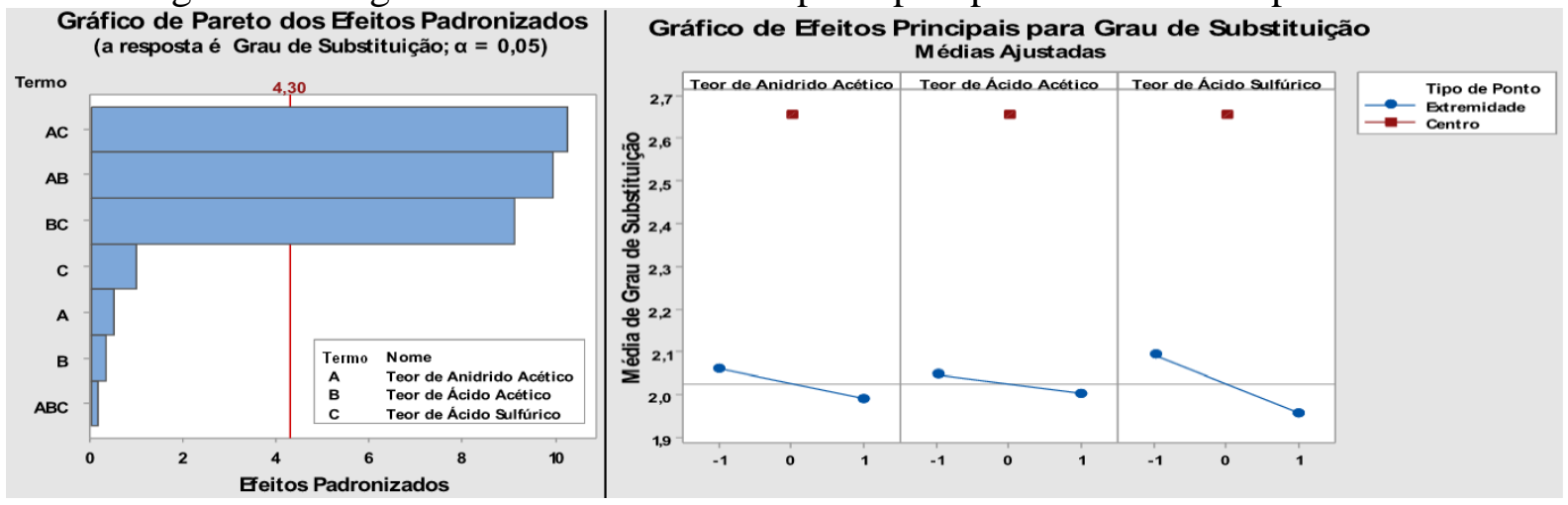

A Tabela 3 mostra os resultados obtidos pelo teste " $t$ " de Student para a eficiência. Como previsto, nenhum dos fatores apresentaram módulo de t-valor maior que 4,30 o que confirma a baixa influência apresentada na Figura 2. Quanto aos resultados de p-valor, observa-se que todos são maiores que 0,05 confirmando o resultado obtido pelo teste anterior.

Tabela 3 - Determinação dos valores de t e p de cada termo para a eficiência

\begin{tabular}{ccccc}
\hline Fatores & Coeficiente & Erro Padrão & t & p-valor \\
\hline Média & 47,5 & 12,6 & 3,76 & 0,064 \\
Teor de $\mathrm{C}_{4} \mathrm{H}_{6} \mathrm{O}_{3}$ & $-10,1$ & 12,6 & $-0,80$ & 0,506 \\
Teor de $\mathrm{CH}_{3} \mathrm{COOH}$ & 3,9 & 12,6 & 0,31 & 0,788 \\
Teor de $\mathrm{H}_{2} \mathrm{SO}_{4}$ & 7,2 & 12,6 & 0,57 & 0,627 \\
Teor de $\mathrm{C}_{4} \mathrm{H}_{6} \mathrm{O}_{3} *$ Teor de $\mathrm{H}_{2} \mathrm{SO}_{4}$ & $-15,8$ & 12,6 & $-1,25$ & 0,337 \\
Teor de $\mathrm{C}_{4} \mathrm{H}_{6} \mathrm{O}_{3} *$ Teor de $\mathrm{H}_{2} \mathrm{SO}_{4}$ & 28,1 & 12,6 & 2,22 & 0,156 \\
Teor de $\mathrm{CH}_{3} \mathrm{COOH} *$ Teor de $\mathrm{H}_{2} \mathrm{SO}_{4}$ & 3,6 & 12,6 & 0,29 & 0,800 \\
\hline
\end{tabular}

Seguindo o mesmo raciocínio, os resultados apresentados na Tabela 4 para a variável resposta GS, demonstram que os efeitos das interações entre os fatores estudados são mais influentes do que o efeito individualizado. $\mathrm{O}$ p-valor das interações de anidrido acético com ácido acético, anidrido acético com ácido sulfúrico e ácido acético com ácido sulfúrico confirmam que esses fatores foram bem ajustados ao modelo. 


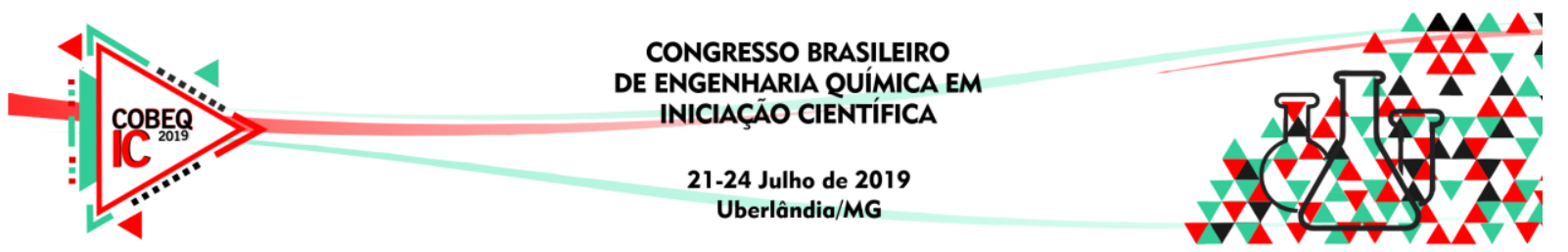

Tabela 4 - Determinação dos valores de t e p de cada termo para a variável resposta GS

\begin{tabular}{|c|c|c|c|c|}
\hline Fatores & Coeficiente & Erro Padrão & $\mathbf{t}$ & p-valor \\
\hline Média & 2,0250 & 0,069 & 29,37 & 0,001 \\
\hline Teor de $\mathrm{C}_{4} \mathrm{H}_{6} \mathrm{O}_{3}$ & $-0,035$ & 0,069 & $-0,51$ & 0,662 \\
\hline Teor de $\mathrm{CH}_{3} \mathrm{COOH}$ & $-0,0225$ & 0,069 & $-0,33$ & 0,775 \\
\hline Teor de $\mathrm{H}_{2} \mathrm{SO}_{4}$ & $-0,0675$ & 0,069 & $-0,98$ & 0,431 \\
\hline Teor de $\mathrm{C}_{4} \mathrm{H}_{6} \mathrm{O}_{3} *$ Teor de $\mathrm{CH}_{3} \mathrm{COOH}$ & $-0,6875$ & 0,069 & $-9,97$ & 0,010 \\
\hline Teor de $\mathrm{C}_{4} \mathrm{H}_{6} \mathrm{O}_{3} *$ Teor de $\mathrm{H}_{2} \mathrm{SO}_{4}$ & 0,7075 & 0,069 & 10,26 & 0,009 \\
\hline Teor de $\mathrm{CH}_{3} \mathrm{COOH} *$ Teor de $\mathrm{H}_{2} \mathrm{SO}_{4}$ & 0,3000 & 0,069 & 9,14 & 0,012 \\
\hline
\end{tabular}

Além disso, os sinais dos coeficientes dos fatores das Tabelas 3 e 4, juntamente com os resultados dos efeitos principais (Figuras 1 e 2) demonstraram o efeito de cada fator para as variáveis resposta no intervalo analisado. Um coeficiente de valor negativo indica que o fator no nível mais baixo do planejamento exploratório resultará em um valor baixo para a variável resposta. Analogamente, um coeficiente de valor positivo indica que o fator no nível mais alto terá maior eficiência na variável resposta.

Quanto à análise de variância (ANOVA) para a variável resposta eficiência (Tabela 5), percebeu-se que tanto para o modelo linear, quanto nas interações de $2^{\mathrm{a}}$ ordem, os fatores não apresentaram significância estatística, visto que os valores de p-valor são maiores que 0,05 .

Tabela 5 - ANOVA para a variável resposta eficiência

\begin{tabular}{|c|c|c|c|c|}
\hline Fonte de Variação & Graus de liberdade & SQ & QM & p-valor \\
\hline Modelo Linear & 8 & 11227,0 & 1403,38 & 0,560 \\
\hline Interação de $2^{\circ}$ Ordem & 3 & 8410,4 & 2803,47 & 0,328 \\
\hline Curvatura & 1 & 13,7 & 13,69 & 0,927 \\
\hline Erro & 2 & 2555,3 & 1277,67 & \\
\hline Total & 10 & 13782,4 & & \\
\hline
\end{tabular}

Contudo, os resultados para a variável resposta GS através da ANOVA (Tabela 6) apresentaram p-valores menores que 0,05 o que demonstra o significativo efeito das variáveis controle, principalmente nas interações de $2^{\mathrm{a}}$ ordem. Observa-se ainda que apesar do modelo linear indicado para esta variável resposta ter significância estatística a 95\% de confiança, um p-valor de 0,041 para a curvatura indica a existência de uma região de máximo ou mínimo que não pode ser detectada pelo modelo em questão. Isto justificaria um novo estudo aplicando um planejamento experimental que forneça um modelo quadrático mais representativo.

Tabela 6 - ANOVA para a variável resposta GS

\begin{tabular}{ccccc}
\hline Fonte de Variação & Graus de liberdade & SQ & QM & p-valor \\
\hline Modelo Linear & 8 & 11,8734 & 765,65 & 0,025 \\
Interação de 2o Ordem & 3 & 10,9609 & 1883,20 & 0,010 \\
Curvatura & 1 & 0,8614 & 448,71 & 0,041 \\
Erro & 2 & 0,0761 & 0,03803 & \\
Total & 10 & 11,9495 & & \\
\hline
\end{tabular}




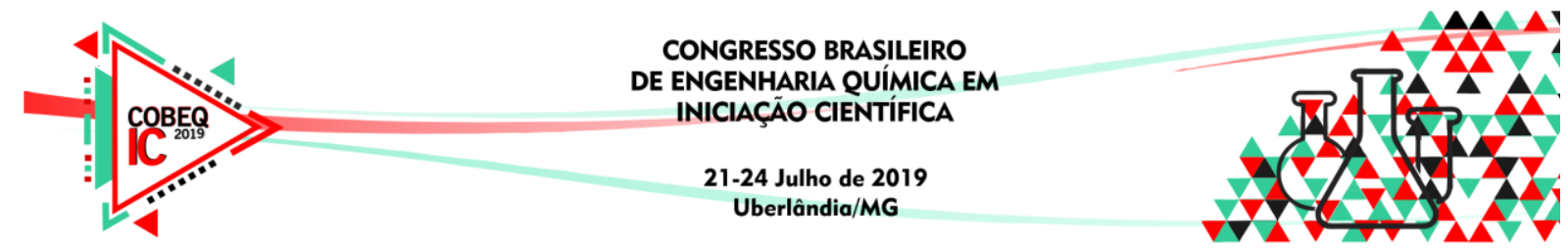

Em resumo, analisando os valores resultantes dos ensaios realizados (Tabela 2), observa-se que a melhor condição experimental estudada foi aquela que utilizou o nível máximo de anidrido acético e mínimos de ácidos acético e sulfúrico, alcançando uma eficiência de 81,2\% e um GS de 2,88. Apesar do ensaio 10 ter apresentado eficiência ainda maior, neste caso o GS se reduziu, distanciando-se do GS típico do triacetato comercial. Vale ressaltar ainda que, o uso de menores quantidades de ácidos, quando analisados no contexto de ampliação de escala, levará a uma redução do consumo de reagentes, o que é sempre positivo no ponto de vista ambiental.

\section{CONCLUSÃO}

A obtenção do acetato de celulose via acetilação homogênea vislumbra viabilidade visto que resultados promissores foram gerados. Contudo, visando o aperfeiçoamento das condições experimentais, estudos subsequentes podem ser realizados utilizando, por exemplo, um novo planejamento estatístico que busque a determinação de um modelo quadrático mais representativo, com a utilização de um delineamento composto central rotacional (DCCR).

\section{REFERÊNCIAS}

ARISTIDOU, A.; PENTTILÄ, M. Metabolic engineering applications to renewable resource utilization. Cur. Op. in Biotech., v.11, p.187-98, 2000.

BARROS NETO, B. de; SCARMINIO, I. S.; BRUNS, R. E. Planejamento e otimização de experimentos, $3^{\mathrm{a}}$ ed. Campinas: UNICAMP, 2007

CANDIDO, Rafael Garcia. Utilização da celulose de resíduos lignocelulósicos para obtenção de produtos de alto valor agregado. 2011. $141 \mathrm{f}$. Tese (Doutorado) - USP, Lorena, 2011.

CHENG, H.n. et al. Synthesis of cellulose acetate from cotton byproducts. Carb. Polym., [s.1.], v. 80, n. 2, p.449-452, abr. 2010.

Companhia Nacional de Abastecimento (CONAB). Disponível em: <http://www.conab.gov.br/>. Acesso em 15 de Março de 2019.

FERREIRA JÚNIOR, M. F. et al. SEM study of the morphology of asymmetric cellulose acetate membranes produced from recycled agro-industrial residues: sugarcane bagasse and mango seeds. Polym. Bul., [s.1.], v. 66, n. 3, p.377-389, 11 jul. 2010.

GALDÁMEZ, E. V. C. Aplicação das técnicas de planejamento e análise de experimentos na melhoria da qualidade de um processo de fabricação de produtos plásticos. 2002. 133 p. Dissertação (Mestrado). Escola de Engenharia de São Carlos, USP, 2002.

PULEO, A. C. et al. The effect of degree of acetylation on gas sorption and transport behavior in cellulose acetate. J. Memb. Sci., p. 301-332. dez. 1989.

SEABRA, J. E. A. Avaliação técnico-econômica de opções para o aproveitamento integral da biomassa de cana no Brasil. Tese (Doutorado) - UNICAMP, Campinas, 2008.

ZUGENMAIER, Peter. Characteristics of cellulose acetates - Characterization and physical properties of cellulose acetate. Macrom. Symp. [s.1.], v. 208, n. 1, p.81-166, mar. 2004. 